\title{
Une série continue de 27 patients adultes opérés d'un spondylolisthésis isthmique L5-S1 par voie combinée : résultats cliniques et radiologiques à 1 an de suivi
}

\section{A continuous series of 27 adult patients treated for L5-S1 isthmic spondylolisthesis by combined approach: Clinical and radiological outcomes at 1 year follow-up}

\author{
N. Koné ${ }^{a},{ }^{*}$, E. Freitas Olim ${ }^{a}$, P. Coloma ${ }^{a}$, A. Rusconi ${ }^{a}$, L. Chenin ${ }^{a}$, O. Sy ${ }^{c}$, \\ I.S. Souaréd ${ }^{d}$ C. Barrey ${ }^{a, b}$ \\ a Département de neurochirurgie et chirurgie du rachis, hôpital P.-Wertheimer, hospices civils de Lyon, université Claude-Bernard Lyon-1, 59, boulevard \\ Pinel, 69003 Lyon, France \\ ${ }^{\mathrm{b}}$ Institut de biomécanique humaine Georges-Charpak, arts et métiers ParisTech, Ensam, 151, boulevard de l'Hôpital, 75013 Paris, France \\ ${ }^{\mathrm{c}}$ Laboratoire d'épidémiologie, recherche clinique et santé communautaire, faculté de médecine et de pharmacie, CHU Hassan II, Km 2.200 route de \\ Sidi-Harazem, 1893 Fès, Maroc \\ d Hôpital de l'Amitié Sino-Guinéenne, Hasigui Kipé-Ratoma 030, 710 Conakry, Guinée
}

Spinal fusion

Spondylolisthesis

Neurosurgical procedures

Lordosis

Sagittal balance
Keywords:

Combined approach

\section{A B S T R A C T}

Through this single-center consecutive prospective study, we evaluated the results of a combined approach for L5-S1 isthmic spondylolisthesis, using a polyetheretherketone (PEEK) interbody lordotic cage during anterior approach and pedicle screw-based posterior fixation. Between 2010 and 2014 , 27 adult patients were treated for L5-S1 isthmic spondylolisthesis (high and low grades) by a combined approach with a minimum follow-up of one year. Clinical outcome was assessed before surgical treatment and at four months and one year after surgery by: VAS, Oswestry Index (ODI) and RollandMorris scores. Two observers evaluated the following radiological parameters: pelvic incidence, pelvic tilt, lumbar lordosis, segmental lordosis L5-S1, anterior and posterior disc height, spinal vertical axis (SVA), SVA/sacro-femoral distance (SFD) ratio. Fusion was evaluated on the CT scan at one-year followup. Blood loss, surgery time and complications were also collected. The mean age was 47.7 years ( \pm 16.9 ). The VAS, ODI and Rolland-Morris scores were significantly improved postoperatively, decreased from 7.5 $( \pm 1.45) ; 48$ ( \pm 19.25$) ; 15.3$ ( \pm 4.67$)$ before the surgery to $3.8( \pm 2.55) ; 28.7( \pm 19.58)$ and $7.76( \pm 7.21)$ respectively at one year after the surgery $(P=0.05)$. The mean follow-up was 3.3 years. Mean surgery time was $193.7 \mathrm{~min}$ ( \pm 37 ). Fusion was obtained in $100 \%$ of cases. Segmental lordosis L5-S1, pelvic tilt, slippage, anterior and posterior L5-S1 disc height were significantly improved postoperatively, they passed from 20.1 ; 22.6 ; $35.3 \% ; 26.4 \% ; 17.9 \%$ to $29.5 ; 20.6 ; 20.3 \% ; 64.4 \% ; 36.3 \%$ respectively. Combined surgical procedure meets the required goals of surgery in the treatment of adults L5-S1 isthmic spondylolisthesis

\section{R É S U M É}

Mots clés :

Arthrodèse vert,brale Spondylolisth,sis Technique neurochirurgicale

Voie combinée

Lordose lombaire

Équilibre sagittal
Cette étude prospective consécutive monocentrique évalue les résultats d'une arthrodèse par voie combinée pour le traitement des spondylolisthésis isthmiques L5-S1, utilisant une cage intersomatique en polyétherétherkétone (PEEK) par voie antérieure et une ostéosynthèse postérieure par vis pédiculaires. Entre 2010 et 2014, 27 patients adultes ont été traités d'un spondylolisthésis isthmique L5-S1 (de haut et de bas grades) par voie combinée avec un suivi minimum d'un an. L'évolution clinique a été évaluée : échelle visuelle analogique (EVA), score d'Oswestry (ODI) et échelle d'incapacité fonctionnelle pour l'évaluation des lombalgies (EIFEL). L'évaluation radiologique faite par deux observateurs était basée sur : 
incidence pelvienne, version pelvienne, lordose lombaire, lordose segmentaire, hauteurs discales, axe vertébral sagittal, ratio spinal vertical axis (SVA)/distance sacro-fémorale (SFD) et sur l'obtention de la fusion par le scanner à un an. Les pertes sanguines, la durée de chirurgie et les complications ont également été recueillies. L'âge moyen était 47,7 ans $( \pm 16,9)$. Les scores EVA, ODI et EIFEL ont été significativement améliorés passant respectivement de 7,58 $( \pm 1,45), 48,00( \pm 19,25), 15,32( \pm 4,67)$ en préopératoire à 3,84 $( \pm 2,55) ; 28,7( \pm 19,58) ; 7,76( \pm 7,21)$ à un an postopératoire $(p=0,05)$. Le recul moyen de la série était de 3,3 ans. La fusion a été constatée dans $100 \%$ des cas. Les hauteurs discales, la lordose segmentaire L5-S1, la version pelvienne, le glissement moyen, les hauteurs discales antérieure et postérieure ont été modifiées de façon significative passant respectivement de $20,1^{\circ} ; 22,6^{\circ} ; 35,3 \% ; 26,4 \% ; 17,9 \%$ à $29,5^{\circ}$; $20,6^{\circ} ; 20,3 \% ; 64,4 \% ; 36,3 \%$ en postopératoire. Cette procédure remplit les objectifs requis de la chirurgie des spondylolisthésis isthmiques L5-S1 de l'adulte.

\section{Introduction}

L'incidence des spondylolyses isthmiques lombaires dans la population adulte est d'environ $6 \%$ [1] et concerne dans plus de $90 \%$ des cas la vertèbre L5 [2]. $[3,4]$.

Elles évolueront dans 60 à $80 \%$ des cas vers un spondylolisthésis

Le traitement chirurgical de référence du spondylolisthésis isthmique (SPLi) reste l'arthrodèse instrumentée de l'étage olisthésique.

Il concerne environ 10 à $20 \%$ des patients et uniquement [5] après l'échec d'un traitement médical, en présence de troubles neurologiques moteurs ou de listhésis progressifs ou supérieurs à 50 $\%$.

La technique chirurgicale optimale pour la réalisation de l'arthrodèse n'est pas consensuelle selon les données de la littérature $[6,7]$.

L'arthrodèse peut tre obtenue via un abord postérieur par arthrodèse postérolatérale (PLF) [8,9] ou intersomatique. L'arthrodèse intersomatique lombaire peut être réalisée par voie postérieure (PLIF) [8,9], par voie transforaminale (TLIF) [10,11] ; par un abord antérieur (ALIF) [12,13] ou par une approche combinée en un seul temps (antérieur + postérieur) [14-19].

Partant de l'hypothèse que l'instabilité de l'étage olisthésique et la discopathie étaient les causes essentielles des lomboradiculalgies, nous avons traité cette pathologie par voie combinée en utilisant une cage intersomatique en polyétherétherkétone (PEEK) remplie de protéines ostéo-inductrices (Bone Morphogenetic Protein-2) par voie antérieure rétropéritonéale en L5-S1 (ALIF) associée à un vissage pédiculaire postérieur.

Le but de cette étude a été d'évaluer les résultats cliniques et radiologiques d'une série prospective et continue de SPLi L5-S1 chez des patients adultes traités par voie combinée.

\section{Matériel et méthode}

Entre janvier 2010 et décembre 2014, 27 patients consécutifs ont été opérés pour un SPLi L5-S1 de haut et de bas grades par voie combinée (antérieure et postérieure).

Un suivi clinique et radiographique prospectif d'un an au minimum a été effectué pour tous les patients. Les patients exclus de l'étude étaient ceux opérés d'un SPLi L5-S1 avec un délai de suivi inférieur à un an et ceux opérés en L5-S1 pour d'autres pathologies (traumatique, tumorale, infectieuse) de la charnière lombosacrée.

\subsection{Technique chirurgicale}

La stratégie chirurgicale était fonction du degré de glissement L5-S1 selon la classification de Meyerding [20]. On procédait initialement par un abord antérieur puis postérieur pour les SPLi de bas grade (I, II), les SPLi de haut grade (III, IV) étaient abordés par voie postérieure puis secondairement par voie antérieure.

L'exploration préopératoire de l'anatomie vasculaire de la région lombosacrée par un angioscanner 3D a été systématiquement réalisée pour étudier les rapports vasculaires prévértebraux avec analyse [21] :

- du niveau de la bifurcation aorto-iliaque et ilio-cave par rapport au disque L5-S1 ;

- de la largeur de la fenêtre vasculaire ;

- de la présence de variations anatomiques ou d'athérosclérose.

L'ALIF L5-S1 était réalisée par voie rétropéritonéale droite classique en utilisant une cage intersomatique en PEEK (Antelis, Scient'x-Alphatec, Carlsbad, États-Unis) remplie de BMP-2 (Bone Morphogenetic Protein-2).

La fixation pédiculaire postérieure L5-S1 ou L4-S1 était réalisée avec des vis mono- et poly-axiales associées à des tiges prélordosées (Antelis, Scient'x-Alphatec, Carlsbad, États-Unis), elle était fonction de l'importance du glissement de L5 sur S1 et de l'état du disque sus-jacent (L4-L5). En cas de sténose symptomatique lombaire avec douleur radiculaire, un récalibrage du canal lombaire latéral était réalisé. L'autogreffe postérieure était systématique par produit d'arthrectomie et des processus épineux. La mise en compression sur les têtes de vis permettait d'optimiser le gain de lordose.

\section{2. Évaluation clinique}

L'évolution clinique a été évaluée en préopératoire, à quatre mois et un an postopératoire par les mesures de l'échelle visuelle analogique (EVA), de l'index d'Oswestry (ODI) et de l'échelle d'incapacité fonctionnelle pour l'évaluation des lombalgies (EIFEL).

\section{3. Évaluation radiologique}

L'évaluation radiologique était basée sur la mesure des paramètres de l'équilibre sagittal sur des clichés de radiographie de la colonne totale et du bassin avec le système EOS (Biospace, Paris, France) [22] en pré- et en postopératoire à un an.

Concernant la lordose lombaire L5-S1, elle a été mesurée entre le plateau sacré et le plateau supérieur de L1.

La lordose segmentaire (Lseg) L5-S1 a été mesurée entre le plateau supérieur de L5 et le plateau supérieur de S1.

Le ratio de spinal vertical axis (SVA)/distance sacro-fémorale (SFD) : a été calculé en rapportant le SVA à la distance sacro-fémorale (c'est-à-dire, la distance horizontale entre l'axe bicoxofémoral et la verticale passant par le coin postéro-supérieur de S1) (Fig. 1). Ce paramètre reflète l'équilibre sagittal global de la colonne vertébrale au-dessus du bassin (valeur normale $-0,9 \pm 1$ ) [23]. 


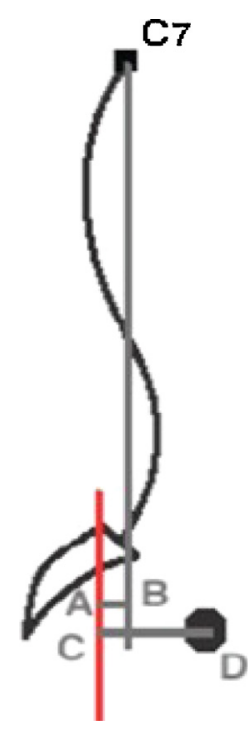

Fig. 1. $C 7$ plumbline/distance sacro-fémorale, ratio [25] $C 7 / S F D=A B / C D$. C7 plumbline/sacro-femoral distance ratio [25] $C 7 / S F D=A B / C D$.

Les hauteurs discales (HD) antérieure et postérieure de l'espace intersomatique (étage olisthésique L5-S1) ont été calculées en faisant rapporter respectivement les hauteurs discales mesurées au niveau des murs vertébraux antérieur et postérieur à la hauteur du mur vertébral postérieur du corps vertébral de L5 (Fig. 2).

Les paramètres rachidiens et pelviens ont été mesurés par deux observateurs, les moyennes ont été relevées avec une différence inférieure ou égale à $5 \%$.

En parallèle, les données peropératoires (pertes sanguines, durée de la chirurgie) et les complications (neurologiques, infectieuses, mécaniques et générales) étaient recueillies durant le suivi.

L'obtention d'une arthrodèse a été évaluée par le scanner de contrôle à un an postopératoire selon les critères définis par le groupe de recherche sur la fusion intervertébrale [24] et la classification de Lenke et al. [25].

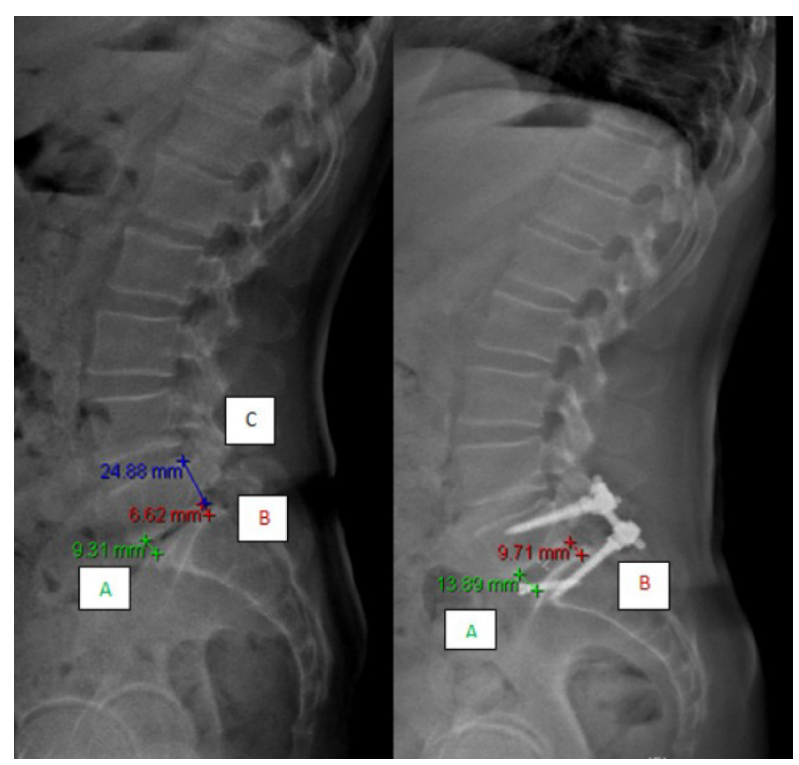

Fig. 2. Hauteurs discales (HD) antérieure et postérieure de l'espace discal L5-S1 (HD antérieure $=\mathrm{A} / \mathrm{C} \times 100 ;$ HD postérieure $=\mathrm{B} / \mathrm{C} \times 100)$.

Anterior and posterior disc space heights $(D H) L 5-S 1$ (DH anterior $=A / C \times 100 ; D H$ posterior $=B / C \times 100)$.

\subsection{Analyse statistique}

Toutes les analyses statistiques ont été réalisées à l'aide du logiciel IBM SPSS Statistics version 20. Un $t$-test a été réalisé afin d'étudier si la différence entre la valeur préopératoire et la valeur postopératoire était significative pour les paramètres cliniques et radiologiques. Une valeur $(p)$ inférieure ou égale à 0,05 était considérée comme significative.

\section{Résultats}

La série comportait 23 femmes et 4 hommes (27 patients) dont l'âge moyen était 47,7 ans $( \pm 16,9)$. Le recul moyen de la série était de 3,3 ans $( \pm 1,6)$. La durée moyenne de l'approche combinée était de $193,7 \mathrm{~min}( \pm 37)$ dont $76,9 \mathrm{~min}( \pm 16,8)$ et $116,7 \mathrm{~min}( \pm 33,6)$ respectivement pour les abords antérieur et postérieur. Les pertes sanguines moyennes durant les deux abords étaient de $285 \mathrm{~mL}$ $( \pm 239,3)$.

\subsection{Résultats cliniques}

Les scores cliniques (EVA, ODI, EIFEL), reportés dans le Tableau 1, étaient significativement améliorés un an après la chirurgie $(p=0,05)$.

À quatre mois postopératoire, seule l'EVA était significativement améliorée $(p=0,05)$.

\subsection{Complications}

Nous avons relevé chez un des patients porteurs d'un SPLi de grade III, une atteinte motrice segmentaire L5 bilatérale partielle postopératoire (avec une force musculaire cotée à 3/5 en postopératoire immédiat ayant récupéré partiellement avec une force motrice à $4 / 5$ à un an) et une infection du site opératoire résolutive sous antibiotiques après lavage parage au bloc opératoire. Nous n'avons pas observé de complications vasculaires, viscérales ou génito-urinaires.

\subsection{Résultats radiologiques}

La répartition en grade de Meyerding (I, II, III, IV) en préopératoire comportait respectivement le nombre de cas suivant par grade : $5 ; 15$ (soit 20 cas de SPLi de bas grade) ; $4 ; 1$ (soit 5 cas de haut grade). Nous avons relevé en postopératoire, 1 grade 0,19 grades I, 5 grades II (soit 25 cas de bas grade) et 2 grades III (soit 2 cas de haut grade).

Les paramètres de l'équilibre sagittal spino-pelvien (SVA, SVA/SFD, LL (lordose lombaire), Lseg, IP (incidence pelvienne), VP (version pelvienne)) ainsi que les HD (antérieure et postérieure) et les degrés de listhésis pré- et postopératoires sont reportés dans le Tableau 2.

Les HD (antérieure et postérieure), la Lseg L5-S1 (Fig. 3), la VP et le glissement de L5 par rapport à S1 ont été modifiées de façon significative en postopératoire.

La LL $(p=0,062)$ n'a pas été modifiée de façon significative tout comme le SVA $(p=0,1)$, et le rapport SVA/SFD $(p=0,12)$.

La réduction du glissement chez les patients atteints de spondylolisthésis de haut grade était de 22,6 \% ; en revanche, elle était de $13,1 \%$ en moyenne pour ceux atteints d'un bas grade.

La chronologie des deux abords chirurgicaux étaient différentes selon le grade (Tableau 3):

- SPLi de bas grade : vingt cas (dans tous les cas, l'abord antérieur a précédé la chirurgie postérieure) ; 
Tableau 1

Évolution des scores cliniques à quatre mois et à un an postopératoire.

Changes in clinical score at 4 months and 1 year postoperatively.

\begin{tabular}{|c|c|c|c|c|c|}
\hline & Préopératoire & Postopératoire (4 mois) & $p$ (4 mois) & Postopératoire (1 an) & $p(1$ an $)$ \\
\hline EVA & $7,58 \pm 1,45$ & $5,42 \pm 2,52$ & $0,005^{*}$ & $3,84 \pm 2,55$ & $0,000^{*}$ \\
\hline ODI & $48,00 \pm 19,25$ & $41,68 \pm 22,64$ & 0,26 & $28,7 \pm 19,58$ & $0,001^{*}$ \\
\hline EIFEL & $15,32 \pm 4,67$ & $12,32 \pm 7,50$ & 0,08 & $7,76 \pm 7,21$ & $0,036^{*}$ \\
\hline
\end{tabular}

EIFEL : échelle d'incapacité fonctionnelle pour l'évaluation des lombalgies ; EVA : échelle visuelle analogique ; ODI : score d'Oswestry ; * $p$ significatif.

Tableau 2

Évolution des paramètres radiologiques.

Evolution of radiological parameters.

\begin{tabular}{|c|c|c|c|c|c|c|c|}
\hline \multirow[b]{2}{*}{ Observateurs } & \multicolumn{3}{|c|}{ Préopératoire } & \multicolumn{3}{|c|}{ Postopératoire } & \multirow[t]{2}{*}{$p$ (t-test) } \\
\hline & OBS1 & OBS2 & Moyenne & OBS1 & OBS2 & Moyenne & \\
\hline Glissement & $35,0 \%$ & $35,6 \%$ & $35,3 \pm 16,9 \%$ & $21,0 \%$ & $21,7 \%$ & $20,3 \pm 13,7 \%$ & $0,000^{*}$ \\
\hline IP & $70,4^{\circ}$ & $71,0^{\circ}$ & $70,7 \pm 11,32^{\circ}$ & $70,4^{\circ}$ & $71,0^{\circ}$ & $70,7 \pm 11,3^{\circ}$ & - \\
\hline VP & $22,7^{\circ}$ & $22,6^{\circ}$ & $22,6 \pm 7,43^{\circ}$ & $20,9^{\circ}$ & $21,2^{\circ}$ & $20,7 \pm 6,6^{\circ}$ & $0,027^{*}$ \\
\hline $\begin{array}{l}\text { LL (L1S1) angle entre le plateau supérieur de L1 } \\
\text { (1ère vertèbre lombaire) et le plateau } \\
\text { supérieur de S1 (1ère vertèbre sacrée) }\end{array}$ & $64,2^{\circ}$ & $65,0^{\circ}$ & $64,6 \pm 7,50^{\circ}$ & $67,6^{\circ}$ & $69,2^{\circ}$ & $68,4 \pm 10,6^{\circ}$ & 0,062 \\
\hline $\begin{array}{l}\text { Lseg (L5S1) angle entre le plateau supérieur de } \\
\text { L5 (5ème vertèbre lombaire) et le plateau } \\
\text { supérieur de S1 (1ère vertèbre sacrée) }\end{array}$ & $20,0^{\circ}$ & $20,2^{\circ}$ & $20,1 \pm 8,10^{\circ}$ & $29,1^{\circ}$ & $30,0^{\circ}$ & $29,5 \pm 9,2^{\circ}$ & $0,000^{*}$ \\
\hline HDA & $24,8 \%$ & $24,4 \%$ & $24,6 \pm 16,24 \%$ & $64,5 \%$ & $64,4 \%$ & $64,4 \pm 12 \%$ & $0,000^{*}$ \\
\hline HDP & $18,5 \%$ & $17,4 \%$ & $17,9 \pm 9,59 \%$ & $36,1 \%$ & $36,6 \%$ & $36,3 \pm 9,3 \%$ & $0,000^{*}$ \\
\hline SVA & $21,8 \mathrm{~mm}$ & $21,7 \mathrm{~mm}$ & $21,7 \pm 27,98 \mathrm{~mm}$ & $15,1 \mathrm{~mm}$ & $14,2 \mathrm{~mm}$ & $14,6 \pm 22,3 \mathrm{~mm}$ & 0,1 \\
\hline SFD & $52,0 \mathrm{~mm}$ & $50,9 \mathrm{~mm}$ & $51,4 \pm 13,45 \mathrm{~mm}$ & $49,0 \mathrm{~mm}$ & $50,9 \mathrm{~mm}$ & $48,98 \pm 13,6 \mathrm{~mm}$ & 0,055 \\
\hline SVA/SFD & 0,39 & 0,38 & $0,39 \pm 0,48$ & 0,30 & 0,27 & $0,28 \pm 0,34$ & 0,12 \\
\hline
\end{tabular}

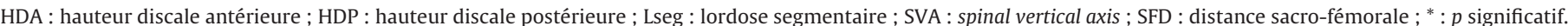

- SPLi de haut grade : cinq cas (dans tous les cas, le temps postérieur a précédé le temps antérieur, excepté un cas de grade III).

Cette étude regroupait 11 instrumentations L5-S1 et 14 instrumentations L4-S1 (Fig. 4).

Le taux de fusion était de $100 \%$ chez 26 patients ayant bénéficié d'un scanner de contrôle (le contrôle TDM n'a pas été effectué chez un des opérés à un an postopératoire (Fig. 5).

\section{Discussion}

Le traitement du spondylolisthésis par lyse isthmique de l'adulte repose, le plus souvent, sur l'arthrodèse instrumentée de l'étage olisthésique. Celle-ci peut être obtenue via un abord postérieur par arthrodèse postérolatérale (PLF) $[8,9]$ ou intersomatique par voie postérieure (PLIF) [8,9], par voie transforaminale (TLIF) $[10,11]$ via un abord antérieur (ALIF) [12,13] ou par une approche combinée, antérieure et postérieure (arthrodèse circonférentielle) [14-19].

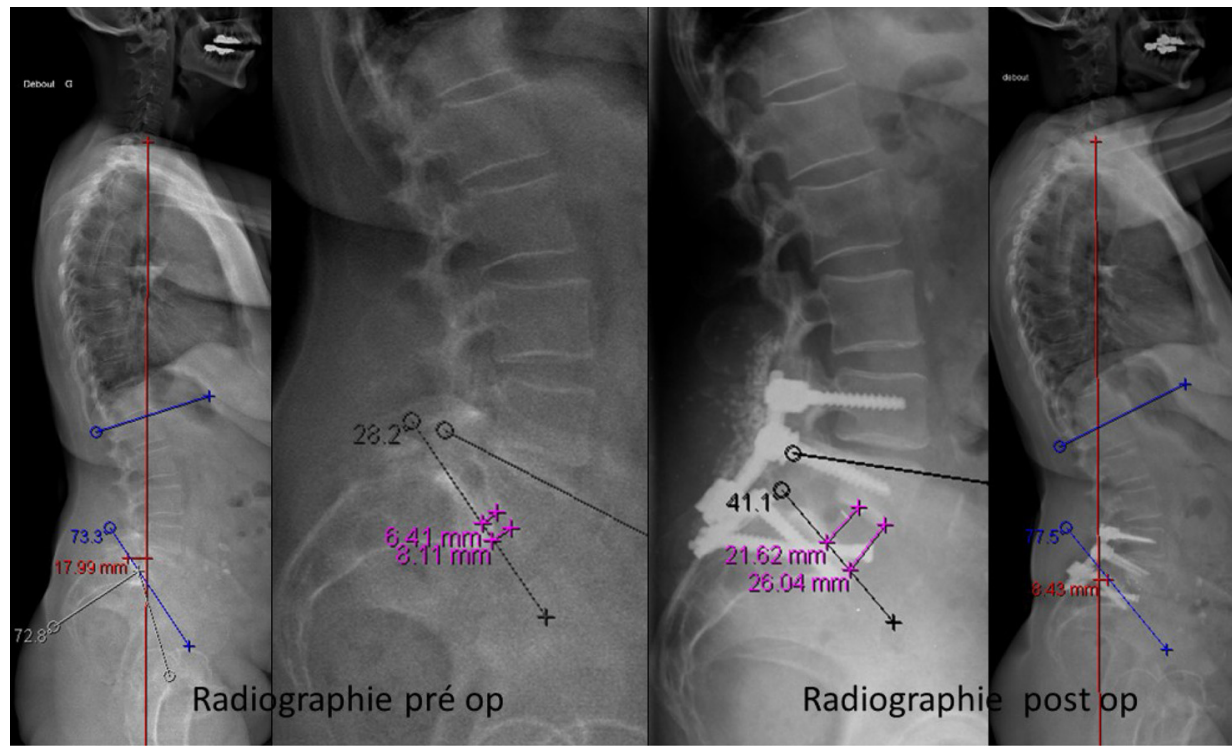

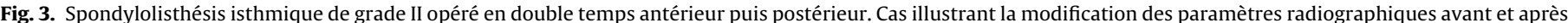

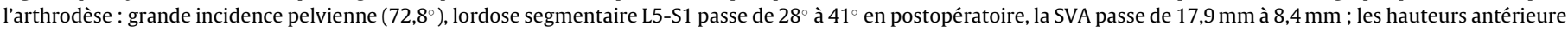
et postérieure de l'espace discal L5-S1 passe respectivement de 8,1 mm;6,4 mm à $26 \mathrm{~mm} ; 21,6 \mathrm{~mm}$.

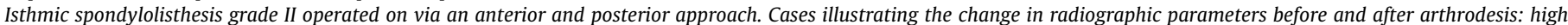

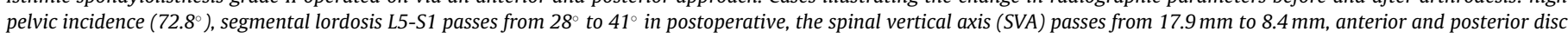
space heights L5-S1 passes respectively from $8.1 \mathrm{~mm} ; 6.4 \mathrm{~mm}$ to $26 \mathrm{~mm} ; 21.6 \mathrm{~mm}$. 


\section{Tableau 3}

Chronologie des abords (antérieur et postérieur) en fonction du degré du spondylolisthésis L5-S1.

Anterior and posterior approach chronology depending on the grade of L5-S1 isthmic spondylolisthesis.

\begin{tabular}{lcl}
\hline \multicolumn{2}{l}{ Chronologies des chirurgies } & \\
\hline Grade avant la chirurgie & Abord antérieur $1^{\text {er }}$ & Abord postérieur $1^{\mathrm{er}}$ \\
\hline I & 5 & - \\
II & 15 & - \\
III & 1 & 3 \\
IV & - & 1 \\
Total & 21 & 4 \\
\hline
\end{tabular}

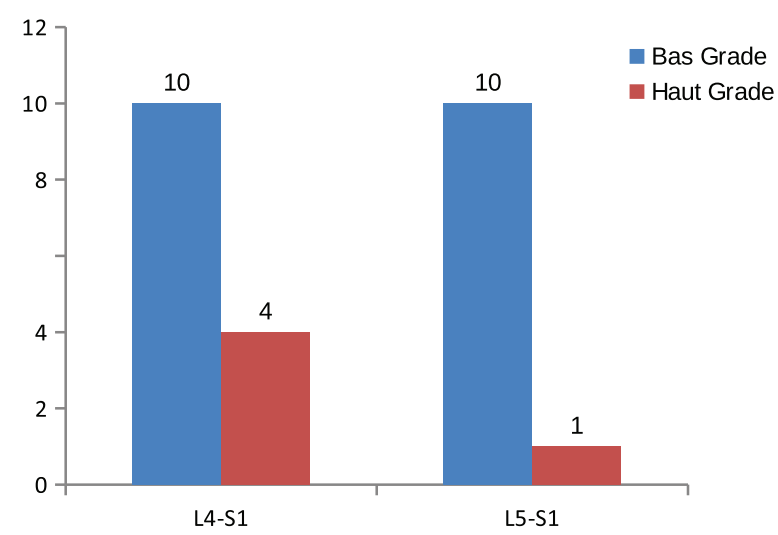

Fig. 4. Nombre de segments fixés par voie postérieure. Number of segments fixed by posterior approach.

Nous avons évalué l'efficacité d'une approche combinée, utilisant une cage intersomatique en PEEK remplie de BMP (Bone Morphogenetic Protein-2) par voie antérieure et des vis pédiculaires postérieures.

Dans une revue récente de la littérature (neuf études comparatives), Wang et al. [7] analysent les résultats radiologiques et fonctionnels des différentes techniques d'arthrodèse pour traiter un spondylolisthésis par lyse isthmique de l'adulte. Ils rapportent respectivement $98 \%, 92 \%, 92 \%, 95,2 \%$ et $82 \%$ de fusion pour les arthrodèses de type ALIF + PLF (fusion postérolatérale), ALIF + PPL (fixation pédiculaire par voie percutanée), TLIF, PLIF et PLF, confirmant l'excellent taux de fusion obtenu par approche combinée.

Gertzbein et al. [19] rapportent un taux de fusion de $97 \%$ suite à une arthrodèse combinée avec une allogreffe antérieure de type ALIF et une fixation postérieure par des vis pédiculaires semirigides.

Barrey et al. [14,15] rapportent $100 \%$ de fusion dans deux études différentes, ce qui concorde avec notre résultat. Ces résultats seraient le produit du regroupement des avantages de la voie antérieure et de la voie postérieure, représentés par :

- pour la voie antérieure : la qualité de la discectomie réalisée, la possibilité d'insérer de larges greffes osseuses ou de larges cages remplies d'ostéo-inducteur de type BMP (Bone Morphogenetic Protein-2) [13,26-28] avec restauration optimale de la lordose ;

- pour la voie postérieure : la qualité biomécanique du montage assurée par la stabilisation pédiculaire [29], la possibilité de réaliser une greffe $360^{\circ}$ et un récalibrage du canal rachidien si nécessaire [30,31].

Bien que l'os autologue reste le matériau de greffe de référence et que le site des crêtes iliaques offre les plus grands volumes disponibles de greffons [32], nous avons utilisé la BMP-2. Dans une étude multicentrique portant sur 279 patients, Burkus et al. [26] retrouvent respectivement un taux moyen de fusion de $94,5 \%$ et $88,7 \%$ en utilisant de la BMP-2 et le greffon osseux autologue prélevé au niveau iliaque pour réaliser une greffe antérieure de type ALIF.

La morbidité au site de prélèvement apparaît comme une réalité certaine avec globalement des troubles sensitifs (douleurs mais aussi hypoesthésies, dysesthésies ou hyperesthésies chroniques) très variables d'une étude à l'autre : $1,2 \%$ pour Younger et Chapman [33] ; 8 \% pour Laurie et al. [34], $27 \%$ pour Hill et al. [32], $32 \%$ pour Burkus et al. [26].

Concernant l'apport de la BMP-2, de nombreux articles montrent un intérêt sur l'obtention de la fusion [13,26-28].

Comme rapportées par certains auteurs [14,35], nous n'avons pas eu de modification significative de l'équilibre global du rachis, ce qui peut, en partie, être expliquée par le fait que la plupart des patients avaient un équilibre rachidien global préopératoire normal. En réalité, dans les SPLi de l'adulte, il existe typiquement un trouble régional de la statique rachidienne avec préservation de l'équilibre global. La correction complète en cas de déséquilibre

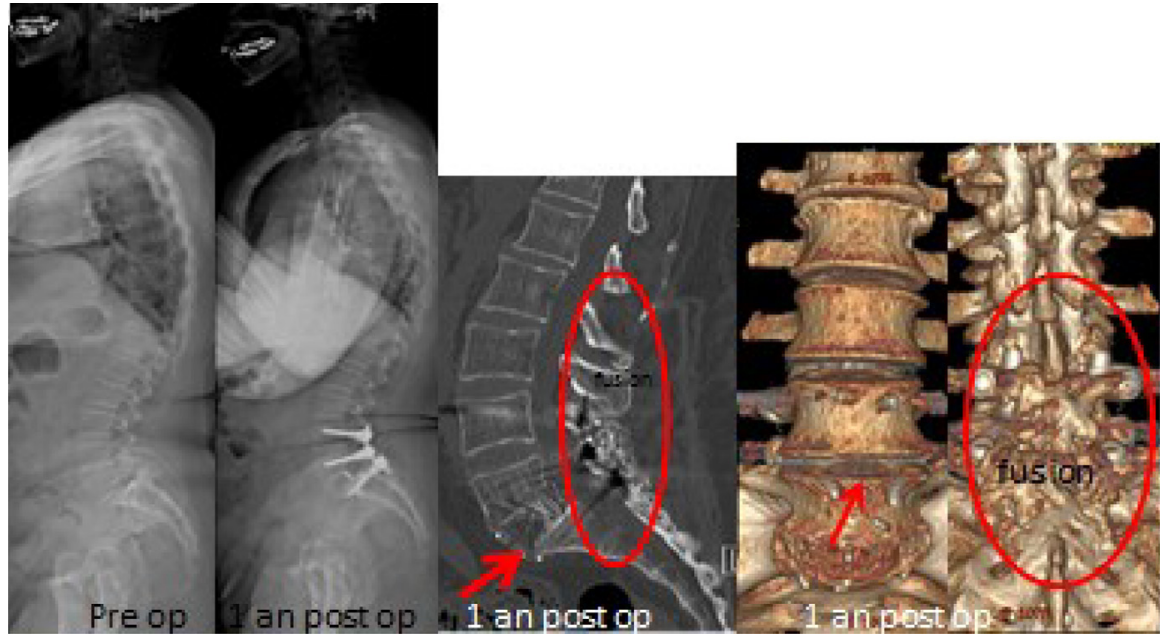

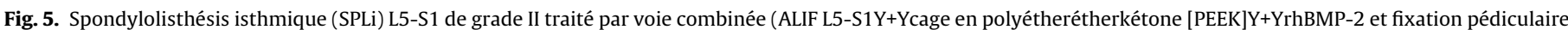
L4-L5-S1Y+Ygreffe postéromédiane). Résultat de la fusion intersomatique et interlamaire à un an postopératoire.

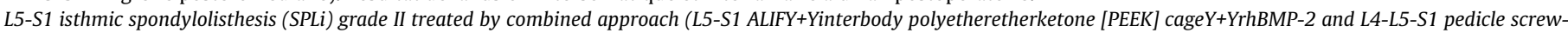
based posterior fixation $Y+Y$ posteromedial bone graft). Interbody and interlaminar fusion results at 1 year postoperative. 
sagittal nécessite des interventions chirurgicales plus invasives, telles que la fusion ou ostéotomies [36] à plusieurs niveaux.

Par ailleurs, la restauration de la hauteur discale et de la lordose segmentaire par la fusion à un ou deux niveaux restent d'une grande importance pour l'amélioration des résultats à court et à long terme $[37,38]$.

Dans une revue récente de la littérature, Wang et al. [7] rapportent que l'ALIF procure de meilleurs résultats que la TLIF et la PLIF dans la restauration de la HD et la LL.

Dans notre étude, la lordose locale L5-S1 et les HD (antérieure et postérieure) ont été modifiées de façon significative $\left(p<10^{-3}\right)$, avec respectivement un gain moyen de $9,46 \pm 8,22^{\circ}, 39,52 \pm 19,71$ $\%, 18,77 \pm 12,97 \%$. En général, la restauration de la lordose segmentaire par d'autres techniques d'arthrodèses intersomatiques (TLIF, PLIF) est limitée seulement de 5-6 ${ }^{\circ}$ [35].

Nous avons constaté une réduction spontanée du glissement de $15 \pm 8,03 \%$ en moyenne, malgré l'absence de la manouvre spécifique de réduction en dehors de l'installation du patient et de la distraction intervertébrale, probablement liée à la curarisation, à la discectomie et la fixation pédiculaire postérieure.

Kim et al. [17,18] et Shim et al. [16] trouvent une réduction spontanée du glissement de 9 à $10 \%$.

$\mathrm{Au}$ vu des résultats fonctionnels que nous avons obtenus, nous concluons que la réduction complète du glissement ne doit pas constituer l'objectif principal mais que l'objectif principal doit être la restauration de la lordose segmentaire adaptée au morphotype.

L'étude de Lian et al. [39] ne montre aucune différence significative entre les résultats des arthrodèses avec réduction et sans réduction pour les spondylolisthésis isthmiques de grade I, II et III.

Il convient de souligner que chez les patients présentant un spondylolisthésis de haut grade et une colonne vertébrale déséquilibrée, la réduction et le réalignement semblent plus importants dans le bénéfice clinique postopératoire [40].

Notre étude regroupait 12 instrumentations L5-S1 et 15 instrumentations L4-S1. L'indication du nombre de segment à fixer postérieurement était fonction de l'état du disque L4-L5 et du degré du SPLi L5-S1.

Vu que dans les SPL de haut grade, l'insertion d'une cage en première intention est très difficile, une instrumentation postérieure L4-S1 semble mieux maintenir la réduction du segment L5-S1. En raison d'une répartition des contraintes en cisaillement sur L4 et L5 [41], nous avons débuté la chirurgie par la voie postérieure dans quatre cas de SPLi de haut grade sur cinq.

Nos résultats concernant la fusion, la restauration des HD et de la Lseg pourraient être dus à l'approche combinée.

Pradhan et al. [42] ont rapporté un taux élevé d'effondrement de greffe et de pseudarthrose suivant des arthrodèses de type ALIF isolée en utilisant l'allogreffe fémorale et la rhBMP-2. La stabilité mécanique de l'instrumentation est d'une importance capitale pour empêcher l'effondrement de la greffe lors du remodelage rapide créé par la rhBMP-2. Par conséquent, une fixation pédiculaire par voie postérieure contribuerait à assurer la réussite d'une fusion lombaire intersomatique, utilisant une greffe osseuse et la rhBMP2 [43]. Le choix d'une fixation postérieure à ciel ouvert par rapport à la voie percutanée nous permet de réaliser une décompression si nécessaire, une greffe postérieure augmentant ainsi les chances de succès de la fusion, en notant que l'ostéosynthèse percutanée représente une alternative intéressante.

Sur le plan clinique, nous avons constaté une baisse significative de l'EVA ainsi qu'une amélioration des scores fonctionnels. Ce qui concorde avec les données de la littérature [7]. Toutefois, il aurait été intéressant d'évaluer la reprise du travail et de mesurer les scores de qualité de vie.

Nous rapportons, dans notre série, un taux de complication (un cas d'infection postopératoire et d'atteinte motrice radiculaire L5 bilatérale) relativement faible par rapport aux séries publiées.
Le taux de complication rapporté par les différentes études est très variable, d'une série à une autre [7].

L'abord combiné regroupe les complications de la voie antérieure et de la voie postérieure. Mobbs et al. [44] retrouvent un taux moyen d'infection de $2,2 \%$ pour les ALIF.

Farrokhi et al. [9] rapportent un taux d'infection de 2,1 \% pour les PLF.

Notre taux d'atteinte neurologique est en dessous de celui rapporté par Ogilvie (3,1\%) [45], ceci pourrait être en corrélation avec le degré de réduction du SPL.

Dans notre étude, la voie combinée était responsable d'un saignement de $285 \pm 239,3 \mathrm{~mL}$, ce qui est largement en dessous du résultat de Pan et al. [10] et de Zaïri et al. [11] utilisant respectivement le TLIF par abord minimal invasif et le TLIF par abord classique.

La limitation de la perte sanguine permet, une diminution de la durée moyenne d'hospitalisation, des complications postopératoires et une récupération rapide [46]. Le faible taux d'infections postopératoires dans notre série pourrait probablement être expliqué par le raccourcissement du temps postérieur par la procédure d'ALIF [47].

D'autres études comparatives seront nécessaires afin d'évaluer cette procédure et démontrer ses avantages par rapport aux autres techniques d'arthrodèses.

\section{Conclusion}

Notre étude a permis de démontrer qu'entre des mains entraînées, cette procédure chirurgicale par voie combinée remplit les objectifs requis de la chirurgie des spondylolisthésis isthmiques L5-S1 de l'adulte en termes de résultats cliniques et radiologiques.

Elle regroupe les avantages de la voie antérieure et ceux de la voie postérieure. Elle devrait être prise en compte par les chirurgiens du rachis devant un spondylolisthésis isthmique L5-S1.

\section{Déclaration de liens d'intérêts}

Les auteurs déclarent ne pas avoir de liens d'intérêts.

\section{Références}

[1] Fredrickson BE, Baker D, McHolick WJ, Yuan HA, Lubicky JP. The natural history of spondylolysis and spondylolisthesis. J Bone Joint Surg Am 1984;66:699-707.

[2] Sakai T, Sairyo K, Suzue N, Kosaka H, Yasui N. Incidence and etiology of lumbar spondylolysis: review of literature. J Orthop Sci 2010;15:281-8.

[3] Toueg CW, Mac-Thiong JM, Grimard G, et al. Prevalence of spondylolisthesis in a population of gymnasts. Stud Health Technol Inform 2010;158:132-7.

[4] Syrmou E, Tsitsopoulos PP, Marinopoulos D, et al. Spondylolysis: a review and reappraisal. Hippokratia 2010;14(1):17-21.

[5] Picault C. Traitement chirurgical. Symposium. Le spondylolisthésis lombosacré. Rev Ortop 1971;57(suppl.):87-162.

[6] Jacobs WC, Vreeling A, De Kleuver M. Fusion for low grade adult isthmic spondylolisthesis: a systematic review of the literature. Eur Spine J 2006;15(4):391-402.

[7] Wang SJ, Han YC, Liu XM, Ma B, Zhao WD, Wu DS, et al. Fusion techniques for adult isthmic spondylolisthesis: a systematic review. Arch Orthop Trauma Surg 2014;134(6):777-84

[8] Ekman P, Möller H, Tullberg T, Neumann P, Hedlund R. Posterior lumbar interbody fusion versus posterolateral fusion in adult isthmic spondylolisthesis. Spine (Phila Pa 1976) 2007;32(20):2178-83.

[9] Farrokhi MR, Rahmanian A, Masoudi MS. Posterolateral versus posterior interbody fusion in isthmic spondylolisthesis. J Neurotrauma 2012;29(8):1567-73.

[10] Pan J, Qian L, Zhou W, Tan J, Zou L, Yang M. Spontaneous slip reduction of lowgrade isthmic spondylolisthesis following circumferential release via bilateral minimally invasive transforaminal lumbar interbody fusion: technical note and short-term outcome. Spine (Phila Pa 1976) 2011;36(4):283-9.

[11] Zaïri F, Thines L, Arikat A, Assaker R. Arthrodèses transforaminales : intérêts de l'abord minimal invasif. Neurochirurgie 2013;59(4-5):171-7.

[12] Riouallon G, Lachaniette $\mathrm{CH}$, Poignard A, Allain J. Outcomes of anterior lumbar interbody fusion in low-grade isthmic spondylolisthesis in adults: a continuous series of 65 cases with an average follow-up of 6,6 years. Orthop Traumatol Surg Res 2013;99(2):155-61. 
[13] Behrbalk E, Uri O, Parks RM, Musson R, Soh RC, Boszczyk BM. Fusion and subsidence rate of stand-alone anterior lumbar interbody fusion using PEEK cage with recombinant human bone morphogenetic protein-2. Eur Spine J 2013:22(12):2869-75.

[14] Barrey C, Boissiere L, D’Acunzi G, Perrin G. One-stage combined lumbo-sacral fusion, by anterior then posterior approach: clinical and radiological results. Eur Spine J 2013;22(Suppl. 6):957-64.

[15] Barrey C, Lucas F, Freitas E, Brandao R, D’Acunzi G, Belliard H, et al. Arthrodèse lombo-sacrée par abord combine, antérieur puis postérieur, en un temps. Le Rachis 2012;1:8-9.

[16] Shim JH, Kim WS, Kim JH, Kim DH, Hwang JH, Park CK. Comparison of instrumented posterolateral fusion versus percutaneous pedicle screw fixation combined with anterior lumbar interbody fusion in elderly patients with L5-S1 isthmic spondylolisthesis and foraminal stenosis. J Neurosurg Spine 2011:15(3):311-9.

[17] Kim JS, Kim DH, Lee SH, Park CK, Hwang JH, Cheh G, et al. Comparison study of the instrumented circumferential fusion with instrumented anterior lumbar interbody fusion as a surgical procedure for adult low-grade isthmic spondylolisthesis. World Neurosurg 2010;73(5):565-71.

[18] Kim JS, Kim DH, Lee SH. Comparison between instrumented Mini-TLIF and instrumented circumferential fusion in adult low-grade lytic spondylolisthesis: can mini-TLIF with PPF replace circumferential fusion? J Korean Neurosurg Soc 2009;45(2):74-80.

[19] Gertzbein SD, Betz R, Clements D, Errico T, Hammerberg K, Robbins S, et al. Semirigid instrumentation in the management of lumbar spinal conditions combined with circumferential fusion. A multicenter study. Spine (Phila Pa 1976) 1996;21(16):1918-25 [discussion 1925-6].

[20] Meyerding HW. Spondylolisthésis. Surg Gynecol Obstet 1932;54:3717.

[21] Barrey C, Ene B, Louis-tisserand G, Montagna P, Perrin G, Simon E. Vascular anatomy in the lumbar spine investigated by 3D CT angiography: the concept of vacular window. World Neurosurg 2013;79(5-6):784-91

[22] Dubousset J, Charpak G, Skalli W, Kalifa G, Lazennec JY. EOS stereo-radiography system: whole-body simultaneous anteroposterior and lateral radiographs with very low radiation dose. Rev Chir Orthop Reparatrice Appar Mot 2007;93:141-3.

[23] Barrey C, Roussouly P, Perrin P, Le Huec JC. Sagittal balance disorders in severe degenerative spine. Can we identify the compensatory mechanisms? Eur Spine J 2011;20(Suppl. 5):626-33.

[24] Burkus JK, Foley K, Haid RW, LeHuec JC, Surgical Interbody Research Group. Radiographic assessment of interbody fusion devices: fusion criteria for anterior lumbar interbody surgery. Neurosurg Focus 2001;10:E11.

[25] Lenke LG, Bridwell KH, Bulis D, Betz RR. Result of in situ fusion for isthmic spondylolisthesis. J Spinal Disord 1992;5:433-42.

[26] Burkus JK, Gornet MF, Dickman CA, Zdeblick TA. Anterior lumbar interbody fusion using rhBMP-2 with tapered interbody cages. J Spinal Disord Tech 2002:15:337-49.

[27] Burkus JK, Transfeldt EE, Kitchel SH, Watkins RG, Balderston RA. Clinical and radiographic outcomes of anterior lumbar interbody fusion using recombinant human bone morphogenetic protein-2. Spine (Phila Pa 1976) 2002;27:2396-408.

[28] Burkus JK, Dorchak JD, Sanders DL. Radiographic assessment of interbody fusion using recombinant human bone morphogenetic protein type 2 . Spine (Phila Pa 1976) 2003:28(4):372-7.

[29] Oxland TR, Lund T. Biomechanics of stand-alone cages and cages in combination with posterior fixation: a literature review. Eur Spine J 2000;9(Suppl. 1):S95-101.
[30] Hsieh PC, Koski TR, O'Shaughnessy BA, Sugrue P, Salehi S, Ondra S, et al. Anterior lumbar interbody fusion in comparison with transforaminal lumbar interbody fusion: implications for the restoration of foraminal height, local disc angle, lumbar lordosis, and sagittal balance. J Neurosurg Spine 2007;7(4): 379-86.

[31] Lee CS, Hwang CJ, Lee DH, Kim YT, Lee HS. Fusion rates of instrumented lumbar spinal arthrodesis according to surgical approach: a systematic review of randomized trials. Clin Orthop Surg 2011;3(1):39-47.

[32] Hill NM, Horne JG, Devane PA. Donor site morbidity in the iliac crest bone graft. Aust N Z J Surg 1999;69:726-8.

[33] Younger EM, Chapman MW. Morbidity a bone graft donor sites. J Orthop Trauma 1989;3(3):192-5.

[34] Laurie SWS, Kaban LB, Mulliken JB, Murray JE. Donor-site morbidity after harvesting rib and iliac bone. J Plast Reconstr Surg 1984;73:933-93.

[35] Ould-Slimane M, Lenoir T, Dauzac C, Rillardon, Hoffmann E, Guigui P, et al Influence of transforaminal lumbar interbody fusion procedures on spinal pelvic parameters of sagittal balance. Eur Spine J 2012;21(6):1200-6.

[36] Drain O, Lenoir T, Dauzac C, Rillardon L, Guigui P. Influence of disc height on outcome of posterolateral fusion. Rev Chir Orthop Reparatrice Appar Mot 2008;94(5):472-80

[37] Liebensteiner MC, Jesacher G, Thaler M, Gstoettner M, Liebensteiner MV, Bach $\mathrm{CM}$. Restoration and preservation of disc height and segmental lordosis with circumferential lumbar fusion: a retrospective analysis of cage versus bone graft. J Spinal Disord Tech 2011;24(1):44-9.

[38] Kumar MN, Baklanov A, Chopin D. Correlation between sagittal plane changes adjacent segment degeneration following lumbar spine fusion. Eur Spine J $2001 ; 10(4): 314-9$

[39] Lian XF, Hou TS, Xu JG, Zeng BF, Zhao J, Liu XK, et al. Single segment of posterior lumbar interbody fusion for adult isthmic spondylolisthesis: reduction or fusion in situ. Eur Spine J 2014;23(1):172-9.

[40] Labelle H, Mac-Thiong JM, Roussouly P. Spino-pelvic sagittal balance of spondylolisthesis: a review and classification. Eur Spine J 2011;20(Suppl 5): 641-6.

[41] Lengert R, Charles YP, Walter A, Schuller S, Godet J, Steib JP. Chirurgie par voie postérieure dans le spondylolisthésis de haut grade. Rev Chir Orthop Traumatol 2014;100(5):358-62.

[42] Pradhan BB, Nassar JA, Delamarter RB, Wang JC. Single-level lumbar spine fusion: a comparison of anterior and posterior approaches. J Spinal Disord Tech 2002:15(5):355-61.

[43] Anderson DG, Sayadipour A, Shelby K, Albert TJ, Vaccaro AR, Weinstein MS Anterior interbody arthrodesis with percutaneous posterior pedicle fixation for degenerative conditions of the lumbar spine. Eur Spine J 2011;20(8): 1323-30.

[44] Mobbs RJ, Phan K, Daly D, Prashanth J, Lennox A. Approach-related complications of anterior lumbar interbody fusion: results of a combined spine and vascular surgical team. Global Spine J 2016;6:147-54.

[45] Ogilvie JW. Complications in spondylolisthesis surgery. Spine 2005;30: S97-101.

[46] Zheng F, Cammisa Jr FP, Sandhu HS, Girardi FP, Khan SN. Factors predicting hospital stay, operative time, blood loss, and transfusion in patients undergoing revision posterior lumbar spine decompression, fusion, and segmental instrumentation. Spine (Phila Pa 1976) 2002;27(8):818-24.

[47] Gruskay J, Kepler C, Smith J, Radcliff K, Vaccaro A. Is surgical case order associated with increased infection rate after spine surgery? Spine (Phila Pa 1976) 2012;37(13):1170-4. 